\title{
Physical model of the contact resistivity of metal-graphene junctions
}

\author{
Ferney A. Chaves, ${ }^{1, a)}$ David Jiménez, ${ }^{1}$ Aron W. Cummings, ${ }^{2}$ and Stephan Roche ${ }^{2,3}$ \\ ${ }^{1}$ Departament d'Enginyeria Electrònica, Escola d'Enginyeria, Universitat Autònoma de Barcelona, \\ Campus UAB, 08193 Bellaterra, Barcelona, Spain \\ ${ }^{2}$ ICN2-Institut Català de Nanociència i Nanotecnologia, Campus UAB, 08193 Bellaterra, Barcelona, Spain \\ ${ }^{3}$ ICREA, Institució Catalana de Recerca i Estudis Avançats, 08070 Barcelona, Spain
}

(Received 15 January 2014; accepted 18 April 2014; published online 30 April 2014)

\begin{abstract}
While graphene-based technology shows great promise for a variety of electronic applications, including radio-frequency devices, the resistance of the metal-graphene contact is a technological bottleneck for the realization of viable graphene electronics. One of the most important factors in determining the resistance of a metal-graphene junction is the contact resistivity. Despite the large number of experimental works that exist in the literature measuring the contact resistivity, a simple model of it is still lacking. In this paper, we present a comprehensive physical model for the contact resistivity of these junctions, based on the Bardeen Transfer Hamiltonian method. This model unveils the role played by different electrical and physical parameters in determining the specific contact resistivity, such as the chemical potential of interaction, the work metal-graphene function difference, and the insulator thickness between the metal and graphene. In addition, our model reveals that the contact resistivity is strongly dependent on the bias voltage across the metal-graphene junction. This model is applicable to a wide variety of graphene-based electronic devices and thus is useful for understanding how to optimize the contact resistance in these systems. (C) 2014 AIP Publishing LLC. [http://dx.doi.org/10.1063/1.4874181]
\end{abstract}

\section{INTRODUCTION}

While graphene has emerged as a promising material for future electronic devices, it is often the metal-graphene (MG) contact resistance that dominates the performance of the device. ${ }^{1}$ For example, although a high carrier mobility of $\sim 10000 \mathrm{~cm}^{2} \mathrm{~V}^{-1} \mathrm{~s}^{-1}$ has been reported on $\mathrm{SiO}_{2},{ }^{2-5}$ the small density of states (DOS) of graphene near the Dirac point can suppress current injection from the metal, resulting in high contact resistivity at the MG interface, which limits the total performance of a graphene transistor. ${ }^{6,7}$ In radio-frequency circuits, the key figure of merit is the maximum frequency of oscillation $f_{\text {max }}$, which is the frequency at which the power gain drops to unity. This parameter turns out to be very sensitive to the contact resistance, especially in the absence of full current saturation. ${ }^{1}$ Therefore, the metal-graphene contact resistance is a critical component of graphene-based devices, and controlling its properties is a prerequisite for device optimization.

In two- or three-terminal semiconductor devices, the contact resistance is commonly described with the transmission line model, ${ }^{8-10} R_{c}=\sqrt{\rho_{c} R_{s}} \operatorname{coth}\left(L / L_{T}\right) / L_{W}$, where $L\left(L_{W}\right)$ is the length (width) of the contact, $R_{s}$ is the semiconductor sheet resistance under the metal, $\rho_{c}$ is the specific contact resistivity of the metal-semiconductor junction, and $L_{T}$ is the transfer length, which is the characteristic length over which current injection occurs between the semiconductor and the metal. In the diffusive regime, this parameter is related to the specific contact resistivity through the relation $L_{T}=\sqrt{\rho_{c} / R_{s}}$, while a more detailed description of $L_{T}$ can be found in the work of Xia et al. ${ }^{11} \mathrm{In}$

${ }^{a)}$ Electronic mail: ferneyalveiro.chaves@uab.cat any case, it is evident that $\rho_{c}$ plays a crucial role in the magnitude and length dependence of the metal-graphene contact resistance.

There have been many experimental studies of the MG junction in the scientific literature. ${ }^{12-16}$ The relevant role played by graphene in metal-semiconductor junctions has also been evidenced by the recent experimental work of Byun et al., ${ }^{17}$ where a true ohmic contact in Ni-Si junctions has been demonstrated by using an interfacial graphene layer to lower the Schottky barrier. In addition, the role of metallic leads in determining the transport properties of graphenebased junctions has been addressed by several theoretical studies. ${ }^{11,18-20}$ However, a direct and comprehensive model of carrier transport between a two-dimensional graphene sheet and a three-dimensional metal is still lacking. Such a model is needed to understand the intrinsic factors that control the magnitude of the specific contact resistivity, and thus to understand how to optimize the graphene-metal contact resistance. The model presented is intended to clarify the relevant physical ingredients that come into play in determining the contact resistivity between the metal and the graphene underneath. However, the additional component of the contact resistance in graphene-based FETs coming from the junction formed between graphene under the metal and the graphene channel has not been considered. A full model taking into account these two components and how they do depend on the gate voltage will be the subject of a future work.

We address this problem by formulating an analytical model of the tunneling current and contact resistivity of the metal-insulator-graphene (MIG) heterostructure, from which the MG junction can be seen as a particular case. Our model is based on the Bardeen Transfer Hamiltonian (BTH) 
(a)



(b)

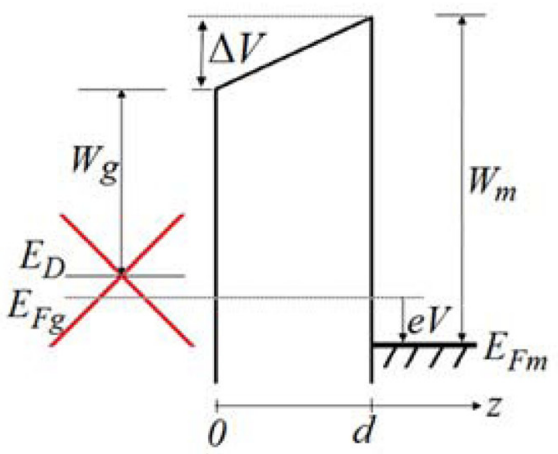

FIG. 1. (a) Physical structure and (b) band diagram of the metal-insulatorgraphene structure examined in this paper. method, ${ }^{21,22}$ which allows us to split the metal-graphene system into separate metal-dipole and dipole-graphene subsystems with known Hamiltonians. In the framework of the BTH method, the probability of elastic tunneling is calculated using Fermi's golden rule. This gives a quantitative estimate of the coupling between the metal and graphene states, allowing us to obtain analytical formulas for both the tunneling current and contact resistivity as a function of the applied voltage. This model allows us to identify the key parameters in determining the metal-graphene contact resistivity and can also be used in larger-scale simulations of graphene-based electronic devices.

\section{THEORY, DISCUSSION, AND RESULTS}

\section{A. Electrostatics}

We start with a description of the electrostatics of the MIG heterostructure. Represented in Fig. 1(a), it consists of a metal (M) electrode treated as an equipotential with voltage $V$ and work function $W_{m}$, and a grounded graphene layer (G) with work function $W_{g}$. These are separated by an intermediate insulator (I) layer with permittivity $\epsilon=\epsilon_{r} \epsilon_{0}$ and thickness $d$, where $\epsilon_{r}$ is the relative permittivity of the insulator and $\epsilon_{0}$ is the permittivity of free space. In the MIG band diagram of Fig. 1(b), $E_{F g}$ is the graphene Fermi energy, $E_{D}$ is its Dirac point energy, and $E_{F m}$ is the metal Fermi energy. An interfacial potential step $\Delta V$ is developed across the (I) layer due to charge transfer and the chemical interaction between the graphene and the metal. ${ }^{23}$
With respect to the Dirac point $E_{D}$, the shift of the graphene Fermi level is defined as $\Delta E=E_{D}-E_{F g}$ and a relationship between the applied voltage $V$ and $\Delta E$ can be obtained from a voltage (potential energy) loop around the MIG band diagram

$$
\Delta E=W_{m}-W_{g}-e \Delta V-e V .
$$

The interfacial potential step $\Delta V$ can be expressed as $\Delta V=\Delta_{t r}+\Delta_{c h}$, where the $\Delta_{t r}$ term results from charge transfer between the (M) and the $(\mathrm{G})$ and the $\Delta_{c h}$ term is the chemical potential describing the short-range interaction from the overlap of the (M) and $(\mathrm{G})$ wavefunctions. According to the model given by Khomyakov et al., ${ }^{24}$ the value of $\Delta_{c h}$ depends strongly on the separation distance $d$ (Fig. 1(b)) and it becomes negligible for $d \gtrsim 4 \mathrm{~nm}$.

To model the electron transfer contribution, $\Delta_{t r}$, we use a planar capacitor model such that $\Delta_{t r}(\Delta E)=z_{d} Q_{n e t} / \epsilon$, where $z_{d}$ represents the effective distance between the charge sheets of (M) and (G) (Fig. 2(b)) and $Q_{n e t}=e(p-n)$ is the net charge sheet density within the graphene. ${ }^{25}$ In addition, we have assumed charge neutrality for the structure (i.e., the electric field goes to zero outside the structure) and thus $Q_{n e t}=-Q_{m}$, where $Q_{m}$ is the surface charge density in the metal. Finally, although in MG junctions the electrodes permit transmission, the reflection probability is nearly unity because the electrons see a barrier height $4 \mathrm{eV}$ and thus the MG junction can be treated as a mesoscopic capacitor. ${ }^{26}$ Substituting the conventional expression ${ }^{24}$ for $p-n$ into Eq. (1) yields the relation (a)

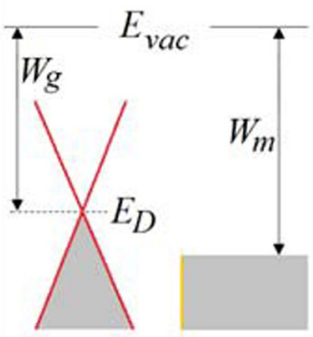

(b)

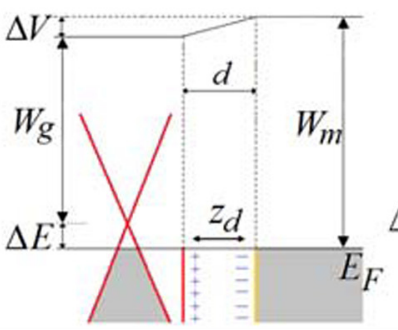

(c)

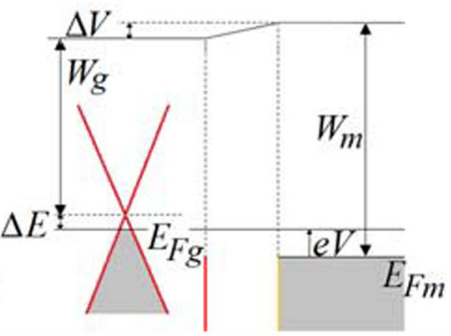

FIG. 2. (a) Band diagram of isolated graphene and metal. (b) Band diagram of a metal-insulator-graphene structure at equilibrium showing charge transfer. A voltage drop $\Delta V$ is produced over the interfacial layer, and $\Delta E$ represents the shift of the graphene Fermi level with respect to the Dirac point. (c) Nonequilibrium band diagram of the metal-insulator-graphene structure. As it is mentioned in the main text, the metal-graphene junction is obtained by letting $d \rightarrow d_{e q}$. 


$$
\alpha f\left(\Delta E / k_{B} T\right)+\Delta E+e V-e V_{D}=0,
$$

where we have defined

$$
e V_{D}=W_{m}-W_{g}-e \Delta_{c h}
$$

The physical meaning of $V_{D}$ is that of the voltage applied to the metal needed to align the graphene Fermi level to the Dirac point. Also, in Eq. (2), $\alpha=2 e^{2} z_{d} /\left(\epsilon \pi \hbar^{2} v_{f}^{2}\right)$ and $f(x)=\left(k_{B} T\right)^{2}\left[F(-x)-F_{1}(x)\right]$, with $F_{1}(x)$ being the FermiDirac integral of order 1. Equation (2) has a closed-form analytical solution at zero temperature and should be solved numerically for $T>0$. As $T \rightarrow 0$, the $f$ function reduces to $f= \pm \Delta E^{2} / 2$, where the upper (lower) sign applies for $\Delta E<0(\Delta E>0)$. Equation (2) then becomes a simple quadratic equation for $\Delta E$ whose solution is

$$
\Delta E= \pm \frac{\sqrt{1+2 \alpha e\left|V-V_{D}\right|}-1}{\alpha} .
$$

In Eq. (4), the upper sign applies for $V \leq V_{D}$ and the lower sign for $V>V_{D}$. This result holds not only for MIG structures but also for MG junction since, as was mentioned previously, a MG junction can be seen as a particular case of the MIG structure where the layer (I) represents a dipole layer with $d_{e q}$ being the equilibrium separation distance between $(\mathrm{M})$ and $(\mathrm{G}){ }^{24,27,28}$ In this work, we model $z_{d}=d-d_{0}$ with $d_{0}=0.24 \mathrm{~nm}$. We have used $d=d_{e q}, \epsilon_{r}=1$, and $\Delta_{c h}>0$ for MG junctions and $d>d_{e q}, \epsilon_{r}=4$, and $\Delta_{c h}=0$ for MIG structures.

In Fig. 3, we show, for $T \rightarrow 0$, the behavior of $\Delta E$ as a function of the voltage $V$ in a MG junction for five different metals. At $V=0$, metals, such as $\mathrm{Cu}, \mathrm{Ag}$, and $\mathrm{Al}$, dope the graphene n-type while $\mathrm{Pt}$ and $\mathrm{Au}$ electrodes result in p-type graphene. For the sake of comparison, we have also shown $\Delta E$ for a hypothetical metal $X$ with work function $W_{m}=W_{g}=4.5 \mathrm{eV}$ and $\Delta_{t r}=0$, so that graphene is undoped at $V=0$. Given the weak dependence of Eq. (2) on the temperature, as $T \approx 300 \mathrm{~K}$, the curves represented in Fig. 3 do not change significantly except for the slopes near $\Delta E=0$. Therefore, Eq. (4) is a very good approximation of Eq. (2).

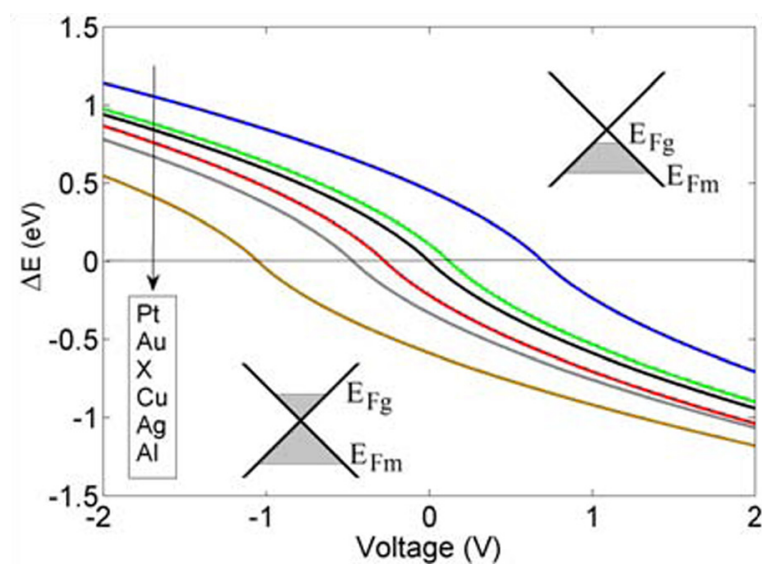

FIG. 3. Graphene Fermi level shift with respect to the Dirac point as a function of the bias voltage $V$ in a metal-graphene junction for different contact metals. The equilibrium separation distances used here are taken from Table I.
For the results shown in Fig. 3, we have not included metals, such as $\mathrm{Co}, \mathrm{Ni}, \mathrm{Pd}$, or $\mathrm{Ti}$, typically used as metallic contacts, because those materials strongly disturb the graphene band structure. In particular, the characteristic linear dispersion of graphene at the K-point is destroyed ${ }^{24}$ and a more detailed model that takes into account the modified graphene band structure would be necessary. ${ }^{29}$ Furthermore, a model for the $\Delta_{c h}$ term has not been established with these metals. However, we can apply our model to these metals in MIG structures, as will be shown later. This is because we assume that the insulator layer does not modify the graphene linear dispersion relation ${ }^{30}$ and the $\Delta_{c h}$ term is considered to be zero. The solutions for Eq. (2) $(\mathrm{T}>0)$ and Eq. (4) $(\mathrm{T}=0)$ will be used later for the calculation of the tunneling current.

\section{B. Current and resistivity}

The specific constant resistivity $\rho_{c}$ of the MG junction is defined as $\rho_{c}=\left.(d J / d V)^{-1}\right|_{V=0}$, where $J$ is the tunneling current density between metal $(\mathrm{M})$ and graphene $(\mathrm{G})$ across the dipole layer and $V$ is the voltage applied to the metal. ${ }^{7,31}$ In the Appendix, we show how to calculate the tunneling current density from the BTH approach, ${ }^{21,22}$ starting from the expression for the tunneling current

$$
I=g_{S} g_{V} \sum_{g, m}\left\{\Gamma_{g m} f_{g}\left(E_{g}\right)\left[1-f_{m}\left(E_{m}\right)\right]-\Gamma_{m g} f_{m}\left(E_{m}\right)\left[1-f_{g}\left(E_{g}\right)\right]\right\},
$$

where both the subscripts $g$ and $m$ label the states in the $(\mathrm{G})$ and $(\mathrm{M})$ electrodes with energies $E_{g}$ and $E_{m}$, respectively; $g_{S}$ is the electron spin degeneracy; $g_{V}$ is the valley degeneracy; and $\Gamma_{g m}$ and $\Gamma_{m g}$ refer to the tunneling rates for electrons moving from $g \rightarrow m$ and $m \rightarrow g$, respectively. Finally, $f_{g}$ and $f_{m}$ are the Fermi occupation factors for the electrons. The tunneling rates are given by the Fermi's golden rule as

$$
\Gamma_{g m}=\frac{2 \pi}{\hbar}\left|M_{g m}\right|^{2} \delta\left(E_{g}-E_{m}\right)=\Gamma_{m g},
$$

where

$$
M_{g m}=\frac{\hbar^{2}}{2 m_{0}} \iint\left(\Psi_{g}^{*} \frac{d \Psi_{m}}{d z}-\Psi_{m} \frac{d \Psi_{g}^{*}}{d z}\right) d S
$$

is the matrix element for the transition, with $m_{0}$ being the effective electron mass in the (I) layer. The terms $\Psi_{g}(\mathbf{r}, z)$ and $\Psi_{m}(\mathbf{r}, z)$ represent the $(\mathrm{G})$ and $(\mathrm{M})$ electron wavefunctions, respectively, and their explicit forms are shown in the Appendix. From the complex exponential dependence of the in-plane wavefunctions, part of the integral of Eq. (7) transforms into the delta-function $\delta\left(\mathbf{k}_{g}-\mathbf{k}_{m}\right)$ when the contact area is large enough, implying the conservation of the in-plane momentum $\mathbf{k}$. On the other hand, the energy delta-function in Eq. (6) guarantees that only energy-conserving tunneling processes are possible.

After some manipulating of Eq. (5), described in the Appendix, Fig. 4(a) shows, at $T=300 \mathrm{~K}$, the magnitude of the tunneling current density of the MG junction as a function of $V$ for $\mathrm{Pt}, \mathrm{Au}, \mathrm{Cu}, \mathrm{Ag}$, and $\mathrm{Al}$ metal electrodes, with 


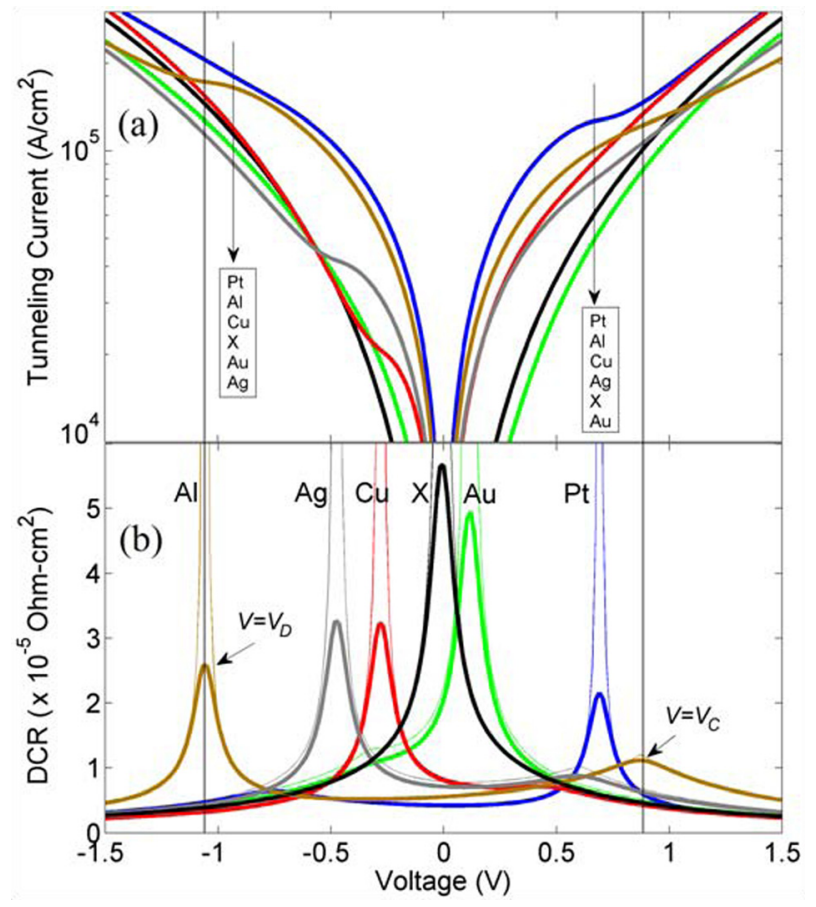

FIG. 4. (a) Tunneling current density $J$ and (b) differential contact resistivity (thick line for $T=300 \mathrm{~K}$ and thin line for $T=0$ ) for the metal-graphene junction as a function of the bias voltage $V$ considering different metal electrodes at the equilibrium separation.

work functions, Fermi energies, and equilibrium separation distance given in Table I. The current-voltage (I-V) relationship of a MG junction or a MIG structure can be understood by considering the possible locations of the metal and graphene Fermi levels around the graphene Dirac point, as illustrated in Fig. 2 assuming $\Delta E>0$ at $V=0$, as is the case for Pt contact. The applied voltage $V$ changes the relative difference between the Fermi levels on each side of the junction according to $E_{F g}-E_{F m}=e V$. If $V>0$, a positive current will flow from the graphene to the metal via tunneling across the (I) layer. A special situation arises when the applied voltage drives the graphene Fermi level to perfect alignment with the Dirac point, resulting in $\Delta E=0$. We have labeled

TABLE I. $W_{m}$ and $d_{e q}$ represent the metal work function and equilibrium separation distance between graphene and the metal, respectively. $E_{F m}$ is the metal Fermi energy at $T=0, \Delta_{c h}$ is the chemical potential of interaction, and $V_{D}$ is the voltage given by Eq. (3).

\begin{tabular}{lccccc}
\hline \hline & $W_{m}(\mathrm{eV})^{\mathrm{a}}$ & $E_{F m}(\mathrm{eV})$ & $d_{e q}(\AA)^{\mathrm{a}}$ & $\Delta_{c h}(\mathrm{eV})^{\mathrm{a}}$ & $V_{D}(\mathrm{~V})$ \\
\hline $\mathrm{Pt}$ & 6.13 & $9.74^{\mathrm{b}}$ & 3.30 & 0.93 & 0.69 \\
$\mathrm{Pd}$ & 5.67 & $7.07^{\mathrm{c}}$ & $\ldots$ & $\ldots$ & $\ldots$ \\
$\mathrm{Au}$ & 5.54 & $5.53^{\mathrm{d}}$ & 3.31 & 0.91 & 0.12 \\
$\mathrm{Cu}$ & 5.22 & $7.00^{\mathrm{d}}$ & 3.26 & 0.99 & -0.27 \\
$\mathrm{Ag}$ & 4.92 & $5.49^{\mathrm{d}}$ & 3.33 & 0.88 & -0.46 \\
$\mathrm{Ti}$ & 4.56 & $4.48^{\mathrm{e}}$ & $\ldots$ & $\ldots$ & $\ldots$ \\
$\mathrm{X}$ & 4.50 & 10.00 & 3.30 & 0.00 & 0.00 \\
$\mathrm{Al}$ & 4.22 & $11.70^{\mathrm{d}}$ & 3.41 & 0.77 & -0.15 \\
\hline \hline
\end{tabular}

${ }^{\mathrm{a}}$ Reference 24.

${ }^{\mathrm{b}}$ Reference 33 .

${ }^{\mathrm{c}}$ Reference 35.

${ }^{\mathrm{d}}$ Reference 34.

e Reference 36. such a bias as $V_{D}$. Analogously, if $V<0$, a negative tunneling current will flow across the (I) layer from the metal to the graphene, and there will be a bias that aligns the metal Fermi level with the graphene Dirac point. We have labeled this bias as $V_{C}$, which occurs when $e V=-\Delta E$. At these critical biases, the low graphene DOS at the Dirac point pinches off the junction and the current changes very little with changes in the applied bias. This can be seen, for example, in the $I-V$ curve of Pt at a bias voltage of $V=0.7 \mathrm{~V}$. Although not shown in Fig. 4(a), the tunneling current at $T=0$ calculated by Eqs. (A12) and (A15)-(A17) (see the Appendix) is barely distinguishable from the results at room temperature. The main difference is the slope of the $I-V$ curve when the applied voltage is $V=V_{D}$. It is due to the change of the quantum capacitance of the graphene. ${ }^{32}$

Figure 4(b) shows the differential contact resistivity, $\operatorname{DCR}(d J / d V)^{-1}$ for a MG junction as a function of bias voltage $V$. The thick lines are for $T=300 \mathrm{~K}$ and thin lines for $T=0$. The DCR exhibits, for every metal, large peaks at voltages $V=V_{D}$ and $V=V_{C}$, which have been labeled for the $\mathrm{Al}$ curve. Metals, such as $\mathrm{Pt}$ and $\mathrm{Au}$, show maximum values of the DCR at $V>0$ while for $\mathrm{Cu}, \mathrm{Ag}$, and $\mathrm{Al}$ the DCR is maximized at $V<0$. For the MG junction with $X$ metal, only one peak appears at $V=0$, similar to Metal-InsulatorMetal (MIM) diodes. ${ }^{37}$ In Fig. 4(b), one can see that a metal, such as $\mathrm{Au}$, leads to a high specific constant resistivity (DCR at $V=0$ ) because its $V_{D}$ value is close to zero, resulting in the Fermi energy close to the Dirac point at zero bias. However, the DCR for Au is significantly lower than that of Pt for $V \sim 0.7 \mathrm{~V}$. Thus, the resistivity of the MG contact is strongly dependent on the voltage drop across the junction. A similar situation occurs for MIG structures, as will be shown later.

Taking advantage of the fact that the specific contact resistivity only weakly depends on the temperature (see Fig. 4(b)), it can be obtained, after some simple algebra, from the expression (A11) of the Appendix

$$
\rho_{c}=\frac{\pi m_{0}^{2} D v_{f}^{2} \hbar^{2} \exp (2 \kappa d)}{8 \sqrt{2} e^{2} m^{3 / 2}}\left(\frac{E_{F m}+E_{\kappa}}{E_{F m} \sqrt{E_{\kappa}}}\right) \frac{1}{\left|\Delta E_{0}\right|},
$$

where $E_{\kappa}=\left(\hbar^{2} / 2 m\right) \kappa^{2}$ and $\Delta E_{0}$ is the shift of the graphene Fermi level with respect to the Dirac at $V=0$. Using the data reported in Table I, Eq. (8) gives the following values for $\rho_{c}$ : $4.59 \times 10^{-6}, 22.44 \times 10^{-6}, 8.53 \times 10^{-6}, 7.9 \times 10^{-6}$, and $5.45 \times 10^{-6} \Omega \mathrm{cm}^{2}$ for $\mathrm{Pt}, \mathrm{Au}, \mathrm{Cu}, \mathrm{Ag}$, and $\mathrm{Al}$, respectively. These values are consistent with experimental results reported by Nagashio and Berdebes. ${ }^{7,15}$ The traditional wisdom is that metals with high or low work functions with respect to pristine graphene make the best contacts because the resulting Fermi level sits far away from the graphene Dirac point. 6,38 However, our model shows that the contact resistivity depends not only on the work function difference but also on the voltage drop across the junction through the $\Delta_{c h}$ term. Although Eq. (8) has been obtained for a MIG structure like that of Fig. 1, it also describes the specific contact resistivity of a graphene-based three-terminal device such as the one studied by Xia et al., when a back gate 


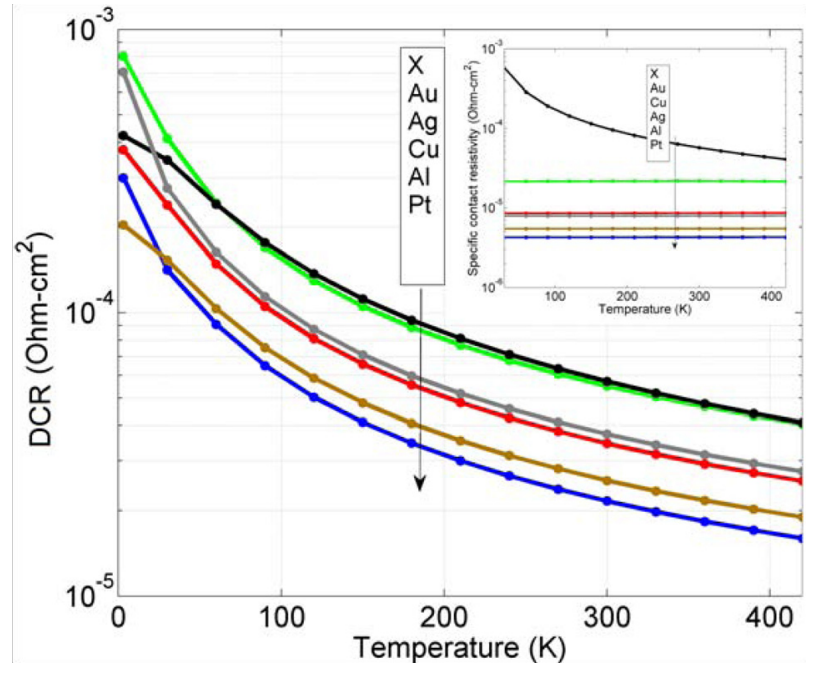

FIG. 5. Differential contact resistivity of the metal-graphene junction at $V=V_{D}$ for different contact metals as a function of the temperature. Inset: specific contact resistivity.

voltage is applied. ${ }^{11}$ However, in the latter case, the value of $\Delta E_{0}$ must be calculated by means of an expression similar to Eq. (2), but taking into account the gate capacitance. Equation (8) permits, in a simple manner, an understanding of the intrinsic factors that control the value of the specific contact resistivity and therefore of the contact resistance. Finally, the temperature dependences of both the DCR at $V=V_{D}$ and the specific contact resistivity $\rho_{c}$ are shown in Fig. 5 and its inset, respectively. Here, like in Fig. 4(b), it is observed that only at $V=V_{D}$ does the DCR depend strongly on the temperature, which is attributed to the reduced DOS in the graphene when $\Delta E=0$, while at $V=0$ that dependence is weak, except to metal with $V_{D} \sim 0$.

Next, we show in Fig. 6 the behavior of the differential tunneling resistivity (DTR) as a function of the voltage $V$ for a MIG structure with $d=0.6 \mathrm{~nm}$. The DTR is calculated in the same manner as the DCR for the MG junction, $(d J / d V)^{-1}$. Here, we have assumed a relative permittivity $\epsilon_{r}=4$ for the (I) layer. In the inset, the expected exponential dependence of the resistivity with $d$ can be observed for a $\mathrm{Pt}$ contact at two different values of $V$. In these structures the DTR depends directly on the work function difference between the metal and graphene since $\Delta_{c h}=0$. It is worth



FIG. 6. DTR of the metal-insulator-graphene heterostructure and its dependence on $d$, assuming $\epsilon=4 \epsilon_{0}$ and $T=300 \mathrm{~K}$. The inset shows the exponential variation of the DTR with $d$ for two different bias voltages. mentioning that, using $\mathrm{Cu}$ or $\mathrm{Ag}$ as the metal electrode, the graphene in the MG junction is n-doped (Fig. 4(b)) but in the MIG structure the graphene is p-doped (Fig. 6). This capability to change the doping type is mediated by the cancelation of chemical potential term $\Delta_{c h}$. In general, when considering a wide range of bias voltages and device geometries, $\mathrm{Pt}$ appears to offer the best performance of the contact metals studied here.

\section{SUMMARY}

In summary, we have developed models for both the tunneling current and the contact resistivity, as a function of the voltage applied to the metal electrode, for the MIG heterostructure based on the BTH method. We have considered the MG junction as a special case of the MIG structure by utilizing a dipole layer as the interfacial layer. From the model for the differential contact resistivity, we have found a simple analytical expression for the specific contact resistivity, which elucidates the role played by the chemical potential of interaction $\Delta_{c h}$, the work function difference, and the insulator thickness between the metal and graphene. In general, the model reveals the role played by the electrical and physical parameters in determining the contact resistivity. Specifically, among the metals considered here, Pt exhibits the smallest specific contact resistivity. However, given the voltage dependence of the contact resistivity, metals like $\mathrm{Au}$ or $\mathrm{Ti}$ can show a smaller resistivity than that of the $\mathrm{Pt}$ depending on the operating regime. The obtained values of the specific contact resistivity are on the order of those given in previous reports. On the other hand, the temperature dependence of the contact resistivity is generally weak at equilibrium for every metal, but is strong and decreases with increasing temperature at $V=V_{D}$, i.e., in the situation corresponding to undoped graphene.

Finally, our model serves as the basis for a more complete model of the contact resistance in graphene-based three-terminal FETs, in which the junction's resistance between the graphene under the metal and the graphene channel should be added. A modified electrostatic model, which takes into account the charge redistribution between metal, graphene and silicon, should be considered to know how the gate voltage impacts on the position of the Fermi energy in respect of the Dirac energy. Overall, our model expounds on the role played by various parameters in determining the contact resistivity of the metal-insulator-graphene junction, which should be useful for optimizing the contact resistance of graphene-based electronic devices. Our model can also be used in larger-scale simulations of these devices.

\section{ACKNOWLEDGMENTS}

We acknowledge support from SAMSUNG within the Global Innovation Program. The research leading to these results has received funding from Ministerio of Economía y Competitividad of Spain under the Project Nos. TEC201231330 and MAT2012-33911, and from the European Union Seventh Framework Programme under Grant Agreement No. 604391 Graphene Flagship. 


\section{APPENDIX: CURRENT TUNNELING CALCULATION}

In this Appendix, we derive an analytical model for the tunneling current density of a MIG structure, relying on the BTH approach. The starting point is Eq. (5) shown in the main text. Then, inserting Eq. (6) into Eq. (5), the tunneling current in the MIG structure can be expressed as

$$
I=g_{V} \frac{4 \pi e}{\hbar} \sum_{g, m}\left|M_{g m}\right|^{2}\left[f_{g}\left(E_{g}\right)-f_{m}\left(E_{m}\right)\right] \delta\left(E_{g}-E_{m}\right) .
$$

Considering the graphene with two identical atoms per unit cell, labeled 1 and 2, the wavefunction for wavevector $\mathbf{k}$ can be written in terms of the basis functions $\Phi_{j \mathbf{k}}(j=1,2)$ on each atom as $\Psi_{g}(\mathbf{r}, z)=\chi_{1}\left(\mathbf{k}_{g}\right) \Phi_{1 \mathbf{k}_{g}}(\mathbf{r}, z)+\chi_{2}\left(\mathbf{k}_{g}\right) \Phi_{2 \mathbf{k}_{g}}(\mathbf{r}, z)$. The basis functions have Bloch form, $\Phi_{j \mathbf{k}_{g}}(\mathbf{r}, z)=\exp$ $\left(i \mathbf{k}_{g} \cdot \mathbf{r}\right) u_{j \mathbf{k}_{g}}(\mathbf{r}, z) / \sqrt{A}$, where $u_{j \mathbf{k}_{g}}(\mathbf{r}, z)$ is a periodic function and $A$ refers to the contact area. These periodic functions are localized around the basis atoms (i.e., as $2 p_{z}$ orbitals) of the graphene, and $u_{j \mathbf{k}_{g}}(\mathbf{r}, z)$ is expected to vary only weakly along the radial coordinate $\mathbf{r}$ in the graphene. Thus, we assume that $u_{j \mathbf{k}_{g}}(\mathbf{r}, z)=f_{\mathbf{j k}_{g}}(\mathbf{r}) g(z)$ and we approximate the radially dependent term $f_{j \mathbf{k}_{p}}(\mathbf{r})$ as numerical constants $f_{1}$ and $f_{2} .{ }^{25}$ The $z$-dependence has the usual decaying form $g(z)=e^{-\kappa z} / \sqrt{D}$, where $\kappa$ is the decay constant of the wavefunction in the barrier, and $D$ is the normalization constant for the z-part of the graphene wavefunction, which we take as equal to an interplane separation in graphite. The decay constant $\kappa$ has the form $\sqrt{2 m \phi / \hbar^{2}+k_{\|}^{2}}$, , where $\phi=5 \mathrm{eV}$ has been taken as the barrier height, and $k_{\|}$is the parallel momentum. For graphene, the latter term is essentially equal to the momentum at the $K$ or $K^{\prime}$ points (i.e., $4 \pi / 3 a$ ) so that $\kappa=20.5 \mathrm{~nm}^{-1}$.

Both $\chi_{1}\left(\mathbf{k}_{g}\right)$ and $\chi_{2}\left(\mathbf{k}_{g}\right)$ have well-known values for graphene in a nearest-neighbor tight-binding approximation ${ }^{39}$

$$
\left[\begin{array}{l}
\chi_{1} \\
\chi_{2}
\end{array}\right]=\frac{1}{\sqrt{2}}\left[\begin{array}{c}
e^{\mp i \vartheta_{\mathbf{k}_{g}} / 2} \\
s e^{ \pm i \vartheta_{\mathbf{k}_{g}} / 2}
\end{array}\right],
$$

where $\vartheta_{\mathbf{k}_{g}}$ is the angle of the relative wavevector, the upper sign is for the band extreme at the $\mathrm{K}$ point of the Brillouin zone and the lower sign is for the $\mathrm{K}^{\prime}$ point, with $s=+1$ for the conduction band (CB) and -1 for the valence band (VB). On the other hand, the metal electrons can be modeled as free incident and reflected particles for $z \geq d$ and with a decaying exponential for $z<d$, namely,

$$
\Psi_{m}(\mathbf{r}, z)= \begin{cases}\frac{e^{i \mathbf{k}_{m} \cdot \mathbf{r}}}{\sqrt{A}} \frac{t e^{k(z-d)}}{\sqrt{D}} & z<d \\ \frac{e^{i \mathbf{k}_{m} \cdot \mathbf{r}}}{\sqrt{A}} \frac{1}{\sqrt{L}}\left[e^{-i k(z-d)}+r e^{i k(z-d)}\right] & z \geq d,\end{cases}
$$

where $t$ and $r$ are the amplitudes of the transmitted and reflected waves, respectively, and $L$ is a normalization constant. As usual, the matching conditions $\left.\Psi_{m}(\mathbf{r}, z)\right|_{z=d^{-}}$ $=\left.\Psi(\mathbf{r}, z)\right|_{z=d^{+}}$and $\left.\left(d \Psi_{m} / d z\right)\right|_{z=d^{-}}=\left.\left(d \Psi_{m} / d z\right)\right|_{z=d^{+}}$have to be fulfilled, resulting in $t=2 \sqrt{(D / L)} k_{z} /\left(k_{z}+i \kappa\right)$. Thus, the matrix elements for the transitions of Eq. (7) can be written as

$$
M_{g m} \approx \frac{\hbar^{2}}{2 m_{0}} \frac{4 k_{z} \kappa}{k_{z}+i \kappa} \Theta\left(\vartheta_{\mathbf{k}_{g}}\right) \frac{e^{-\kappa d}}{\sqrt{L \cdot D}} \frac{1}{A} \int d S e^{i\left(\mathbf{k}_{g}-\mathbf{k}_{m}\right) \cdot \mathbf{r}},
$$

where we have defined $\Theta\left(\vartheta_{\mathbf{k}_{g}}\right)=\chi_{1}^{\star} f_{1}^{\star}+\chi_{2}^{\star} f_{2}^{\star}$. The integral on the right-hand side of Eq. (A4) approaches the deltafunction $\delta\left(\mathbf{k}_{g}-\mathbf{k}_{m}\right)$ when $A \rightarrow \infty$, implying the conservation of in-plane momentum $\mathbf{k}:\left|M_{g m}\right|^{2} \propto\left|A^{-1} \int d S e^{i\left(\mathbf{k}_{g}-\mathbf{k}_{m}\right) \cdot \mathbf{r}}\right|^{2}$ $\rightarrow \delta_{\mathbf{k}_{g}, \mathbf{k}_{m}}^{2}=\delta_{\mathbf{k}_{g}, \mathbf{k}_{m}}$. Incorporating Eq. (A4) into Eq. (A1), we get the following expression for the current

$$
\begin{aligned}
I= & \frac{8 \pi e}{\hbar}\left(\frac{\hbar^{2}}{2 m_{0}} \frac{4 \kappa e^{-\kappa d}}{\sqrt{D}}\right)^{2} \frac{1}{L} \sum_{\mathbf{k}_{g}, \mathbf{k}_{m}, k_{z}}|\Theta(\vartheta)|^{2} \frac{k_{z}^{2}}{k_{z}^{2}+\kappa^{2}} \\
& \times\left[f_{g}\left(E_{g}\right)-f_{m}\left(E_{m}\right)\right] \delta\left(E_{g}-E_{m}\right) \delta_{\mathbf{k}_{g}, \mathbf{k}_{m}} .
\end{aligned}
$$

In deriving Eq. (A5), we have incorporated both the graphene and metal dispersion relations, namely, $E_{g}=E_{g}\left(\mathbf{k}_{g}\right)$ $=E_{D} \pm \hbar v_{f}|k|$ and $E_{m}=E_{m}\left(\mathbf{k}_{m}, k_{z}\right)=\hbar^{2}\left(k_{m}^{2}+k_{z}^{2}\right) / 2 m$, which we have sketched in Fig. 7 for convenience. Considering Eq. (A5) in the limit of large $A, \mathbf{k}_{g}=\mathbf{k}_{m} \equiv k$ where $|\mathbf{k}|=k$, and the equation for the tunneling current becomes

$$
\begin{aligned}
I= & \frac{8 \pi e}{\hbar}\left(\frac{\hbar^{2}}{2 m_{0}} \frac{4 \kappa e^{-\kappa d}}{\sqrt{D}}\right)^{2} \frac{1}{L} \sum_{\mathbf{k}, k_{z}}|\Theta(\vartheta)|^{2} \omega\left(k_{z}\right) \\
& \times\left[f_{g}\left(E_{g}\right)-f_{m}\left(E_{m}\right)\right] \delta\left(E_{g}-E_{m}\right),
\end{aligned}
$$

where we have defined the function $\omega\left(k_{z}\right)=k_{z}^{2} /\left(k_{z}^{2}+\kappa^{2}\right)$. The discrete sums over $k$ and $k_{z}$ are converted to integrals using the recipes $\sum_{\mathbf{k}} \rightarrow \mathrm{A} /(2 \pi)^{2} \iint d \vartheta k d k$ and $\sum_{k_{z}} \rightarrow L / 2 \pi \int d k_{z}$. Integrating the function $|\Theta(\vartheta)|^{2}=\left|f_{1}\right|^{2}+s f_{1}^{\star} f_{2}^{\star} \cos (\vartheta)$ over $\vartheta$ and after some algebra, the tunnel current density becomes

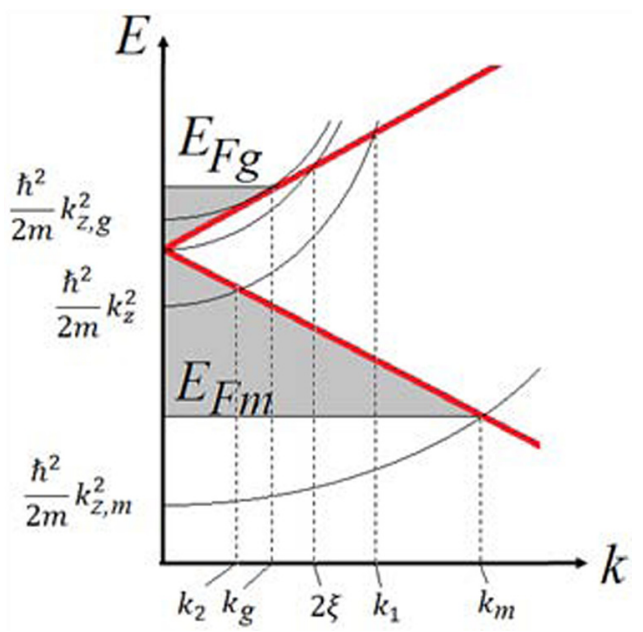

FIG. 7. Parabolic and linear dispersion relations corresponding to metal and graphene electrodes, respectively. At $T=0$, only states lying in the shaded region contribute to the tunneling current. For a given $k_{z}$, both the in-plane momentum $k$ and the total energy $E$ are conserved only for the states with in-plane momentum $k_{1}$ in the conduction band and $k_{2}$ in the valance band. 


$$
J=\eta(\kappa) \iint d k_{z} d k \omega\left(k_{z}\right) k\left[f_{g}\left(E_{g}\right)-f_{m}\left(E_{m}\right)\right] \delta\left(E_{g}-E_{m}\right),
$$

where $\eta(\kappa)=\frac{8 \pi e}{\hbar}\left(\frac{\hbar^{2}}{2 m_{0}} \frac{4 \kappa e^{-\kappa d}}{\sqrt{D}} \frac{\left|f_{1}\right|}{2 \pi}\right)^{2}$ and $\left|f_{1}\right|^{2}$ is a constant of order unity assumed to have no dependence on $k$. The deltafunction in Eq. (A7) guarantees that only processes conserving the energy are possible (Fermi's golden rule). The energy difference appearing in the delta-function can be written as a quadratic equation for $k$,

$$
E_{g}-E_{m}=\frac{\hbar^{2}}{2 m} k^{2} \mp \hbar v_{f} k+\frac{\hbar^{2}}{2 m} k_{z}^{2}-E_{D}=0,
$$

with solutions

$$
k_{1}=\xi+\sqrt{\xi^{2}+k_{D}^{2}-k_{z}^{2}}, \quad k_{2}=\mp \xi \pm \sqrt{\xi^{2}+k_{D}^{2}-k_{z}^{2}} .
$$

The upper (lower) sign of $k_{2}$ applies to the valence (conduction) band and we have defined the constants $\xi=m v_{f} / \hbar$ and $k_{D}^{2}=2 m / \hbar^{2} E_{D}$. Physically, $k_{1}$ and $k_{2}$ are the in-plane momentum $k$ values satisfying both the in-plane momentum and the energy conservation conditions (Fig. 7). In terms of the momentum $k$, the delta-function can be written as $\delta\left(E_{g}-E_{m}\right)$ $=2 m / \hbar^{2} \delta\left[\left(k-k_{1}\right)\left(k-k_{2}\right)\right]$ and an analytic expression for the tunneling current density at $T=0$ can be found

$$
J=\eta(\kappa) \frac{2 m}{\hbar^{2}} \int_{k_{z, m}}^{k_{z, g}} d k_{z} \omega\left(k_{z}\right) \int_{\min \left(k_{g}, k_{m}\right)}^{\max \left(k_{g}, k_{m}\right)} k d k \delta\left[\left(k-k_{1}\right)\left(k-k_{2}\right)\right],
$$

where the meaning of $k_{z, g}, k_{z, m}, k_{g}, k_{m}, k_{1}$, and $k_{2}$ have been graphically represented in Fig. 7. It is worth noting that at $T=0$ only states with energies $E \in\left[E_{F m}, E_{F g}\right]$ contribute to the tunneling current. The energy associated with $k_{1}$ is normally outside of the energy range for which the graphene dispersion relation is lineal $(\sim \pm 1 \mathrm{eV}$, corresponding to $V \sim \pm 2 \mathrm{~V})$, so using the Dirac delta function properties we can write $\Delta\left[\left(k-k_{1}\right)\left(k-k_{2}\right)\right]=\Delta\left(k-k_{2}\right) /\left|k-k_{1}\right|$ and Eq. (A10) becomes

$$
J=\eta(\kappa) \frac{2 m}{\hbar^{2}} \int_{k_{z, m}}^{k_{z, g}} d k_{z} \omega\left(k_{z}\right) \frac{k_{2}\left(k_{z}\right)}{\left|k_{2}\left(k_{z}\right)-k_{1}\left(k_{z}\right)\right|},
$$

where, from Eq. (A9), we have $\left|k_{2}\left(k_{z}\right)-k_{1}\left(k_{z}\right)\right|=2 \xi$ or $\left|k_{2}\left(k_{z}\right)-k_{1}\left(k_{z}\right)\right|=\sqrt{\xi^{2}}+k_{D}^{2}-k_{z}^{2}$ depending on the position of $k_{2}$ (VB or $\mathrm{CB}$ ). For instance, if we consider the situation where $e V_{D} \geq 0$ and $\Delta E<0$, corresponding to Figs. 2(b) and $2(\mathrm{c})$, the range $\left[k_{z, m}, k_{z, g}\right]$ can be split as $\left[k_{z, m}, k_{D}\right] \cup\left[k_{D}, k_{z, g}\right]$, giving the expression

$$
\begin{aligned}
J= & \eta(\kappa) \frac{2 m}{\hbar^{2}}\left\{\int_{k_{z, m}}^{k_{D}} d k_{z} \omega\left(k_{z}\right) \frac{-\xi+R\left(k_{z}\right)}{2 \xi}\right. \\
& \left.+\int_{k_{D}}^{k_{z, g}} d k_{z} \omega\left(k_{z}\right) \frac{\xi-R\left(k_{z}\right)}{2 R\left(k_{z}\right)}\right\},
\end{aligned}
$$

where we have defined $R\left(k_{z}\right)=\sqrt{\xi^{2}+k_{D}^{2}-k_{z}^{2}}$ for convenience, and $k_{z, m}$ and $k_{z, g}$ can be written as

$$
\begin{aligned}
& k_{z, m}=\sqrt{k_{D}^{2}-2 k_{m} \xi-k_{m}^{2}}, \\
& k_{z, g}=\sqrt{k_{D}^{2}+2 k_{g} \xi-k_{g}^{2}},
\end{aligned}
$$

where $k_{m}=(e V+\Delta E) / \hbar v_{f}$ and $k_{g}=|\Delta E| / \hbar v$. Working out Eq. (A12), the following types of integrals appear, which can be solved analytically:

$$
\begin{gathered}
\int d k_{z} \omega\left(k_{z}\right)=k_{z}-\kappa \arctan \left(\frac{k_{z}}{\kappa}\right), \\
\int d k_{z} \omega\left(k_{z}\right) R\left(k_{z}\right)=\frac{1}{2}\left(q^{2}+\kappa^{2}\right) \arctan \left(\frac{k_{z}}{R\left(k_{z}\right)}\right) \\
-\kappa q \arctan \left(\frac{k_{z} q}{\kappa R\left(k_{z}\right)}\right)+\frac{1}{2} k_{z} R\left(k_{z}\right), \\
\int d k_{z} \frac{\omega\left(k_{z}\right)}{R\left(k_{z}\right)}=\arctan \left(\frac{k_{z}}{R\left(k_{z}\right)}\right)-\frac{\kappa}{q} \arctan \left(\frac{k_{z} q}{\kappa R\left(k_{z}\right)}\right),
\end{gathered}
$$

where we have defined $q=\sqrt{\xi^{2}+k_{D}^{2}+\kappa^{2}}$. Similar expressions can be found for the cases $\Delta E>0$ with $V>0$ and for $V<0$. Then, Eq. (A12) can be used to generate the I-V curves of the MIG heterojunction for a variety of different contact configurations.

${ }^{1}$ F. Schwierz, "Graphene transistors: Status, prospects, and problems," Proc. IEEE 101, 1567-1584 (2013).

${ }^{2}$ K. S. Novoselov, A. K. Geim, S. V. Mozorov, D. Jiang, Y. Zhang, S. V. Dubonos, I. V. Grigorieva, and A. A. Firsov, "Electric field effect in atomically thin carbon films," Science 306, 666 (2004).

${ }^{3}$ K. I. Bolotin, K. J. Sikes, Z. Jiang, M. Klima, G. Fudenberg, J. Hone, P. Kim, and H. L. Stormer, "Ultrahigh electron mobility in suspended graphene," Solid State Commun. 146, 351 (2008).

${ }^{4} \mathrm{H}$. Cao et al., "Large-scale graphitic thin films synthesized on $\mathrm{Ni}$ and transferred to insulators: Structural and electronic properties," J. Appl. Phys. 107, 044310 (2010).

${ }^{5}$ H. Cao, Q. Yu, L. A. Jauregui, J. Tian, W. Wu, Z. Liu, R. Jalilian, D. K. Benjamin, Z. Jiang, J. Bao, S. S. Pei, and Y. P. Chen, "Electronic transport in chemical vapor deposited graphene synthesized on $\mathrm{Cu}$ : Quantum Hall effect and weak localization," Appl. Phys. Lett. 96, 122106 (2010).

${ }^{6} \mathrm{~K}$. Nagashio and A. Toriumi, "Density-of-states limited contact resistance in graphene field-effect transistors," Jpn. J. Appl. Phys., Part 1 50, 070108 (2011).

${ }^{7}$ D. Berdebes, T. Low, Y. Sui, J. Appenzeller, and M. Lundstrom, "Substrate gating of contact resistance in graphene transistors," IEEE Trans. Electron Devices 58, 3925 (2011).

${ }^{8}$ D. K. Schroder, Semiconductor Material and Device Characterization, 3rd ed. (John Wiley and Sons, Inc., Hoboken, NJ, 2006).

${ }^{9}$ F. Léonard and A. A. Talin, "Electrical contacts to one- and twodimensional nanomaterials," Nat. Nanotechnol. 6, 773 (2011).

${ }^{10}$ G. K. Reeves and H. B. Harrison, "Obtaining the specific contact resistance from transmission line model measurements," IEEE Electron Device Lett. 3, 111-113 (1982).

${ }^{11}$ F. Xia, V. Perebeinos, Y. Lin, Y. Wu, and P. Avouris, "The origins and limits of metal-graphene junction resistance," Nat. Nanotechnol. 6, 179 (2011).

${ }^{12}$ J. S. Moon et al., "Ultra-low resistance ohmic contacts in graphene field effect transistors,” Appl. Phys. Lett. 100, 203512 (2012).

${ }^{13}$ J. Knoch, Z. Chen, and J. Appenzeller, "Properties of metal-graphene contacts," IEEE Trans. Nanotechnol. 11, 513 (2012). 
${ }^{14}$ A. Venugopal, L. Colombo, and E. M. Vogel, "Contact resistance in few and multilayer graphene devices,” Appl. Phys. Lett. 96, 013512 (2010).

${ }^{15}$ K. Nagashio, T. Nishimura, K. Kita, and A. Toriumi, "Contact resistivity and current flow path at metal/graphene contact," Appl. Phys. Lett. 97, 143514 (2010).

${ }^{16}$ J. Lee et al., "Clean transfer of graphene and its effect on contact resistance," Appl. Phys. Lett. 103, 103104 (2013).

${ }^{17}$ K.-E. Byun et al., "Graphene for true ohmic contact at metal-semiconductor junctions," ACS Nano Lett. 13(9), 4001-4005 (2013).

${ }^{18}$ Q. Ran, M. Gao, X. Guan, Y. Wang, and Z. Yu, "First-principles investigation on bonding formation and electronic structure of metal-graphene contacts," Appl. Phys. Lett. 94, 103511 (2009).

${ }^{19} \mathrm{~N}$. Nemec, D. Tománek, and G. Cuniberti, "Modeling extended contacts to nanotube and graphene devices," Phys. Rev. B 77, 125420 (2008).

${ }^{20}$ S. Barraza-Lopez, M. Vanević, M. Kindermann, and M. Y. Chou, "Effects of metallic contacts on electron transport through graphene," Phys. Rev. Lett. 104, 076807 (2010).

${ }^{21}$ J. Bardeen, "Tunnelling from a many-particle point of view," Phys. Rev. Lett. 6, 57 (1961)

${ }^{22}$ J. Tersoff and D. R. Hamann, "Theory of the scanning tunneling microscope," Phys. Rev. B 31, 805 (1985).

${ }^{23}$ J. G. Champlain, "A first principles theoretical examination of graphenebased field effect transistors," J. Appl. Phys. 109, 084515 (2011).

${ }^{24}$ P. A. Khomyakov, G. Giovannetti, P. C. Rusu, G. Brocks, J. van den Brink, and P. J. Kelly, "First-principles study of the interaction and charge transfer between graphene and metals," Phys. Rev. B 79, 195425 (2009).

${ }^{25}$ R. M. Feenstra, D. Jena, and G. Gu, "Single-particle tunneling in doped graphene-insulator-graphene junctions," J. Appl. Phys. 111, 043711 (2012).

${ }^{26} \mathrm{~T}$. Christen and M. Büttiker, "Low frequency admittance of a quantum point contact,” Phys. Rev. Lett. 77, 144 (1996).
${ }^{27}$ C. Gong, G. Lee, B. Shan, E. M. Vogel, R. M. Wallace, and K. Cho, "First-principles study of metal-graphene interfaces," J. Appl. Phys. 108, 123711 (2010).

${ }^{28}$ K. T. Chan, J. B. Neaton, and M. L. Cohen, "First-principles study of metal adatom adsorption on graphene," Phys. Rev. B 77, 235430 (2008).

${ }^{29}$ N. V. Do and H. A. Le, "Transport characteristics of graphene-metal interfaces," Appl. Phys. Lett. 101, 161605 (2012).

${ }^{30} \mathrm{R}$. Yan et al., "Determination of graphene work function and graphene-insulator-semiconductor band alignment by internal photoemission spectroscopy,” Appl. Phys. Lett. 101, 022105 (2012).

${ }^{31}$ A. M. Roy, J.-Y. Jason Lin, and K. C. Saraswat, "Specific contact resistivity of tunnel barrier contacts used for fermi level depinning," IEEE Electron Device Lett. 31, 1077 (2010).

${ }^{32}$ J. Xia, F. Cheng, J. Li, and N. Tao, "Measurement of the quantum capacitance of graphene," Nat. Nanotechnol. 4, 505 (2009).

${ }^{33}$ E. Gharibshahi and S. Elias, "Influence of dose on particle size and optical properties of colloidal platinum nanoparticles," Int. J. Mol. Sci. 13, 14723-14741 (2012).

${ }^{34}$ N. W. Ashcroft and N. D. Mermin, Solid State Physics (Saunders, 1976).

${ }^{35}$ F. M. Mueller, "Electronic structure of palladium," Phys. Rev. B 1, 4617 (1970).

${ }^{36}$ E. H. Hygh and R. M. Welch, Phys. Rev. B. 1, 2424 (1970).

${ }^{37} \mathrm{~K}$. Choi et al., "A focused asymmetric metal-insulator-metal tunneling diode: Fabrication, DC characteristics and RF rectification analysis," IEEE Trans. Electron Devices 58, 3519 (2011).

${ }^{38}$ B. Huard, N. Stander, J. A. Sulpizio, and D. Goldhaber-Gordon, "Evidence of the role of contacts on the observed electron-hole asymmetry in graphene,” Phys. Rev. B 78, 121402(R) (2008).

${ }^{39}$ N. H. Shon and T. Ando, "Quantum transport in two-dimensional graphite system,” J. Phys. Soc. Jpn. 67, 2421 (1998). 